\title{
Endophilin Drives the Fast Mode of Vesicle Retrieval in a Ribbon Synapse
}

\author{
Artur Llobet, ${ }^{1 \star}$ Jennifer L. Gallop, ${ }^{1 \star}$ Jemima J. E. Burden, ${ }^{2}$ Gamze Çamdere, ${ }^{1}$ Priya Chandra,,${ }^{1}$ Yvonne Vallis, ${ }^{1}$ \\ Colin R. Hopkins, ${ }^{2}$ Leon Lagnado, ${ }^{1}$ and Harvey T. McMahon ${ }^{1}$ \\ ${ }^{1}$ Medical Research Council (MRC) Laboratory of Molecular Biology, Cambridge CB2 0QH, United Kingdom, and 2Department of Biological Sciences, \\ Imperial College, London SW7 2AS, United Kingdom
}

Compensatory endocytosis of exocytosed membrane and recycling of synaptic vesicle components is essential for sustained synaptic transmission at nerve terminals. At the ribbon-type synapse of retinal bipolar cells, manipulations expected to inhibit the interactions of the clathrin adaptor protein complex (AP2) affect only the slow phase of endocytosis $(\tau=10-15 \mathrm{~s})$, leading to the conclusion that fast endocytosis $(\tau=1-2 \mathrm{~s}$ ) occurs by a mechanism that differs from the classical pathway of clathrin-coated vesicle retrieval from the plasma membrane. Here we investigate the role of endophilin in endocytosis at this ribbon synapse. Endophilin A1 is a synaptically enriched $\mathrm{N}$-BAR domain-containing protein, suggested to function in clathrin-mediated endocytosis. Internal dialysis of the synaptic terminal with dominant-negative endophilin A1 lacking its linker and Src homology 3 (SH3) domain inhibited the fast mode of endocytosis, while slow endocytosis continued. Dialysis of a peptide that binds endophilin SH3 domain also decreased fast retrieval. Electron microscopy indicated that fast endocytosis occurred by retrieval of small vesicles in most instances. These results indicate that endophilin is involved in fast retrieval of synaptic vesicles occurring by a mechanism that can be distinguished from the classical pathway involving clathrin-AP2 interactions.

\section{Introduction}

A major role for clathrin-mediated endocytosis in synaptic vesicle retrieval has been confirmed in several functionally and structurally distinct types of synaptic terminals, and in multiple species (Wu et al., 2007). Clathrin-independent modes of retrieval have also been proposed, particularly, fast "kiss-and-run" of small vesicles (Ceccarelli et al., 1973; He et al., 2006), and "bulk retrieval" of larger membrane compartments (Holt et al., 2003; Kasprowicz et al., 2008). The evidence for two kinetically distinct modes of synaptic vesicle retrieval is particularly strong at the ribbon-type synapse of retinal bipolar cells isolated from the retina of goldfish, where the large size of the terminal allows the kinetics of endocytosis to be monitored directly using the capacitance technique (von Gersdorff and Matthews, 1994; Neves and Lagnado, 1999). Manipulations expected to inhibit specific molecular interactions in the clathrin adaptor protein complex (AP2) and

Received Dec. 15, 2009; revised Feb. 18, 2011; accepted March 28, 2011.

Author contributions: C.R.H., L.L., and H.T.M. designed research; A.L., J.L.G., J.J.E.B., G.C., P.C., and Y.V. performed research; A.L., J.L.G., J.J.E.B., and L.L. analyzed data; A.L., J.L.G., L.L., and H.T.M. wrote the paper.

The work was supported by the Medical Research Council (MRC) UK. J.L.G. was a recipient of an MRC Predoctoral Fellowship and a Karn Fund Postdoctoral Fellowship. We thank members of our groups for helpful discussion, in particular, Gary Doherty and Rohit Mittal.

${ }^{*}$ A.L. and J.L.G. contributed equally to the work.

Correspondence should be addressed to either Leon Lagnado or Harvey T. McMahon, MRC Laboratory of Molecular Biology, Hills Road, Cambridge, CB2 0QH, UK. E-mail: II1@mrc-Imb.cam.ac.uk or hmm@mrc-Imb.cam.ac.uk.

A. Llobet's present address: Laboratori Neurobiologia, Institut d'Investigació Biomèdica de Bellvitge (IDIBELL), 08907 L'Hospitalet de Llobregat, Spain.

J. L. Gallop's present address: Harvard Medical School, Department of Systems Biology, 200 Longwood Avenue, Boston, MA 02115.

J.J.E. Burden's present address: MRC Cell Biology Unit, UCL, Gower Street, London WC1E 6BT, UK.

P. Chandra's present address: Babraham Institute, Babraham, Cambridge CB22 3AT, UK.

DOI:10.1523/JNEUROSCI.6223-09.2011

Copyright $\odot 2011$ the authors $\quad 0270-6474 / 11 / 318512-08 \$ 15.00 / 0$ other molecular interactions of the clathrin-dependent pathway only affect the slow phase of retrieval $(\tau=10-15 \mathrm{~s})$, leading to the conclusion that fast endocytosis $(\tau=1-2 \mathrm{~s})$ occurs by a mechanism that is at least partially, if not wholly, distinct (Jockusch et al., 2005). Morphologically, large endosome-like compartments have been seen in bipolar cell terminals after long depolarizations (i.e., tens of seconds) (Holt et al., 2003), and it has been suggested that direct retrieval of membrane into these larger compartments could give rise to faster kinetics of retrieval (LoGiudice and Matthews, 2007).

Here we investigate the potential role of endophilin in vesicle retrieval at the ribbon synapse of retinal bipolar cells. The importance of endophilin at other synapses has been appreciated from studies of null mutants in Caenorhabditis elegans and Drosophila melanogaster, and by acute dialysis of peptides and protein domains into lamprey giant axons, where it is proposed to be involved in the clathrin pathway (Ringstad et al., 1999; Gad et al., 2000; Guichet et al., 2002; Rikhy et al., 2002; Verstreken et al., 2002; Fabian-Fine et al., 2003; Schuske et al., 2003; Dickman et al., 2005). However, these studies do not provide a consistent picture as to the precise functions of endophilin. In C. elegans endophilin mutants, there are increased numbers of all types of clathrin-coated membrane profiles. In contrast, in D. melanogaster, increases in early budding profiles are observed at the neuromuscular junction, and clathrin-coated vesicles are observed in retinal neurons. These discrepancies might be the result of differences in the fixation procedures but might also reflect a general upregulation of the clathrin pathway in response to endophilin deficiency, rather than the inhibition that is commonly supposed. Molecular studies have cast doubt on the significance of endophilin as part of the AP2/clathrin endocytic machinery (Gallop et al., 2006). Endophilin has also been 
implicated in fast retrieval of the vesicular glutamate transporter, albeit via a proposed AP2 pathway (Voglmaier et al., 2006). Using electron microscopy to visualize retrieved compartments and the capacitance technique to make real-time measurements of endocytosis, we find that fast endocytosis at the ribbon synapse of bipolar cells occurs via small, synaptic vesicle-sized compartments and that endophilin is an obligatory effector molecule in this pathway.

\section{Materials and Methods}

Capacitance measurements in bipolar cells. Bipolar cells were isolated from the retina of goldfish by enzymatic digestion and plated onto glass coverslips coated with poly-L-lysine (Neves and Lagnado, 1999). Whole-cell patch-clamp capacitance recordings were performed at room temperature. The Ringer's (bath) solution contained the following (in mM): 120 $\mathrm{NaCl}, 2.5 \mathrm{KCl}, 1 \mathrm{MgCl}_{2}, 2.5 \mathrm{CaCl}_{2}, 10$ glucose, 10 HEPES, pH 7.3, 280 $\mathrm{mOsm} \cdot \mathrm{kg}^{-1}$. The intracellular solution contained the following (in $\mathrm{mM}$ ): 110 Cs methane-sulfonate, 10 tetraethylammonium chloride, 5 $\mathrm{MgCl}_{2}, 3 \mathrm{Na}_{2}$ ATP, $1 \mathrm{Na}_{2}$ GTP, 20 HEPES, 0.4 BAPTA, pH 7.2, 260 $\mathrm{mOsm} \cdot \mathrm{kg}^{-1}$. Protein domains were diluted into the intracellular solution to the indicated final concentrations. In all experiments the series resistance ranged from 10 to $15 \mathrm{M} \Omega$.

Correlative electrophysiology and electron microscopy. Depolarizing bipolar cells isolated as above were plated onto gridded glass coverslips coated with poly-L-lysine. Cells were bathed in low-calcium Ringer's solution $\left(0.2 \mathrm{mM} \mathrm{CaCl}_{2}\right)$ which, after patching, was exchanged with Ringer's solution $\left(2.5 \mathrm{mM} \mathrm{CaCl}_{2}\right)$ containing $10 \mathrm{mg} / \mathrm{ml}$ horseradish peroxidase (HRP). After 2-3 min equilibration, the patched cells were subjected to a train of five, $20 \mathrm{~ms}$ stimulations with 10 s intervals, before being fixed with $2 \%$ PFA $1.5 \%$ glutaraldehyde in buffer A (in mM: 25 HEPES, 38 aspartate, 38 glutamate, 38 gluconate, $2.5 \mathrm{MgCl}_{2}$, 2 EGTA, pH 7.2). Fixative was delivered immediately to the stimulated cells by an electronically controlled pipette positioned directly over the patched cells (for method details, see Rosenmund and Stevens, 1997). Images of the patched cells and their neighboring cells were captured to aid identification of the studied cells in the electron microscope. The fixed cells were treated with diaminobenzidine (DAB) to crosslink the HRP, before being postfixed with $1 \%$ osmium tetroxide $/ 1.5 \%$ potassium ferricyanide and stained with tannic acid. The cells were dehydrated and embedded in Epon stubs, as described previously (Stinchcombe et al., 1995). Coverslips were removed by immersion in liquid nitrogen before serial sectioning, and ultrathin sections were cut en-face using a Reichert-Jung Ultracut E microtome. Sections were stained with lead citrate before being viewed in a Philips CM12 electron microscope. The mean diffusion coefficient of vesicles in the synaptic terminal of bipolar cells has been measured to be $\sim 1.5 \times 10^{-2} \mu \mathrm{m}^{2} \mathrm{~s}^{-1}$ (Holt et al., 2004), and since the average distance from the membrane was only $106 \mathrm{~nm}$, fixation must have occurred within $1 \mathrm{~s}$ of budding from the plasma membrane (see also distribution of distances in Fig. $1 I$ ).

Fitting of capacitance traces. The basic approach to describing the recovery phase of capacitance traces using single- or double-exponential functions has been described previously (Neves and Lagnado, 1999; Jockusch et al., 2005). Three basic situations arose in the present study. In the first, all the excess membrane was recovered in one phase, which could be described as a single exponential (e.g., recovery after $20 \mathrm{~ms}$ or $2 \mathrm{~s}$ stimuli-see black traces in Fig. $4 B, C$ ). In the second, all the excess membrane was retrieved, but recovery was biphasic and was best described as the sum of two declining exponentials (e.g., recovery after a 100 ms stimulus-see black trace in Fig. $4 A$ ). In the third, a proportion of the membrane was not recovered and endocytosis appeared to have stopped within our recording episode (e.g., recovery in the presence of the DNF peptide-purple trace in supplemental Fig. S2, available at www.jneurosci.org as supplemental material). In this last situation, we found that the time course of recovery for that proportion of membrane that was retrieved could also be described adequately as a single exponential.

The decision between single- and double-exponential fits was made by visual inspection followed by comparison of residuals. We set two further criteria before accepting the need for a double exponential over a single: the two rate constants differing by $>4$-fold and the amplitude of the slow phase being at least $10 \%$. The first criterion applied in most situations, because the fast phase typically had a time constant of $\sim 1 \mathrm{~s}$, and the slow phase a time constant of $10 \mathrm{~s}$ or more. For instance, in Figure $4 B(20 \mathrm{~ms}$ stimulus, endophilin $\Delta \mathrm{SH} 3$ ), the fast and slow rate constants differed by a factor of 20. The second criterion was set because an estimate of the slower rate constant was overly sensitive to slight drift in the traces when the amplitude of the slow phase was very small relative to the fast phase. In such situations, only the rate constant and amplitude of the fast phase was recorded, together with the amplitude of the slow phase; the slow rate constant was not deemed reliable. A borderline case is shown by the control response to a $20 \mathrm{~ms}$ stimulus in Figure $4 B$, where the fit suggests that the amplitude of the slow component is $13 \%$ of the total and $k_{\text {slow }}=$ $0.25 \mathrm{~s}^{-1}$. This is an average of 14 individual responses, but when fitting the individual responses to this brief stimulus, a double exponential was used in only one case.

Some capacitance recordings were affected by drift in the absence of stimulation, probably reflecting slow changes in the area of the pipette shank in contact with the solution in the bath. The capacitance was therefore measured for a period of $5 \mathrm{~s}$ before application of a stimulus, and any linear drift was corrected by fitting a straight line to this portion of the trace.

Constructs, cosedimentation assays, and liposome assays. SH3 (Src homology 3) domains [rat endophilin A1 (residues 294-353), rat amphiphysin 1 (residues 604-683), rat amphiphysin 2 (residues 495-588), rat pacsin 2 (residues 407-487), human intersectin 1 SH3 domains A (737-816), C (1000-1066), and E (residues 1155-1220)] were cloned into ER1/Not 1 sites of pGex 4T2, and proteins were expressed in bacteria (BL21 DE3 pLysS) and bound to glutathione Sepharose beads according to standard procedures. One rat brain was homogenized in $4 \mathrm{ml}$ of buffer (50 mм HEPES, pH 7.4, $150 \mathrm{~mm} \mathrm{NaCl}, 4$ mм DTT, 0.1\% Triton X-100; Calbiochem Protease Inhibitor Set III) and extract was made by centrifugation at $100,000 \times g$ for $1 \mathrm{~h}$ at $4^{\circ} \mathrm{C}$. GST-fusion proteins $(50 \mu \mathrm{g})$ were bound to beads and added to $0.5 \mathrm{ml}$ of brain extract, in the presence of peptides at the appropriate concentration. After incubation at $4^{\circ} \mathrm{C}$ for $40 \mathrm{~min}$, beads were washed three times with buffer, including peptides in the first wash. Samples were resuspended in sample buffer and run by SDS-PAGE.

GST full-length rat endophilin A1, GST rat endophilin $\Delta$ SH3 domain (residues 1-247), and dAmphiphysin $\Delta$ SH3 (residues 1-245) were cloned into ER1/Not1 sites of pGex4T2, and proteins were thrombin cleaved before purification by anion exchange and gel filtration. Rat synaptojanin 145 (gift from Peter Parker, Imperial College, London, UK) was cloned into the Notl site of $\mathrm{pBac} 4 \mathrm{x} 1$ with a hexahistidine tag at the $\mathrm{C}$ terminus. Protein was purified on Ni-NTA agarose (Qiagen) followed by S200 gel filtration. Folch Fraction I (Sigma) liposomes in $150 \mathrm{~mm} \mathrm{NaCl}$, $20 \mathrm{~mm}$ HEPES, pH 7.4, were extruded 11 times through polycarbonate membranes (Avanti) to achieve the desired diameter. For tubulation assays, typically $1 \mathrm{mg} / \mathrm{ml}$ liposomes $(200 \mathrm{~nm}$ ) were incubated for $10 \mathrm{~min}$ with 10,20 , and $40 \mu \mathrm{M}$ protein. Samples were spread on electron microscopy grids and stained using 5\% uranyl acetate. (For details, see http:// www.endocytosis.org/techniqs/techniqs.htm.) For the synaptojanin recruitment assays, synaptojanin and full-length endophilin were used at a concentration of $3 \mu \mathrm{M}$ with $0.2 \mathrm{mg} / \mathrm{ml}$ Folch liposomes $(400 \mathrm{~nm}$ ). Endophilin N-BAR domain was used at 1, 2, 5, 10, and $20 \mu \mathrm{M}$. The protein mixture was incubated for $5 \mathrm{~min}$ and sedimented at $100,000 \times g$ for $15 \mathrm{~min}$. Antibodies against endophilin A1 and against synaptojanin were raised in rabbits against Endo1 SH3 domain (Ra74) and full-length synaptojanin (Ra59), respectively. For the Alexa 488 labeling of endophilin, we used the C108S, A247C mutant, and labeled it using Alexa 488 C5-maleimide (Invitrogen).

\section{Results}

\section{The fast mode of endocytosis retrieves small vesicles}

It has been suggested that bulk retrieval into compartments larger than synaptic vesicles (endosomes) may be the dominant mechanism of vesicular membrane recycling in the ribbon synapse of retinal bipolar cells (Paillart et al., 2003). To investigate whether 

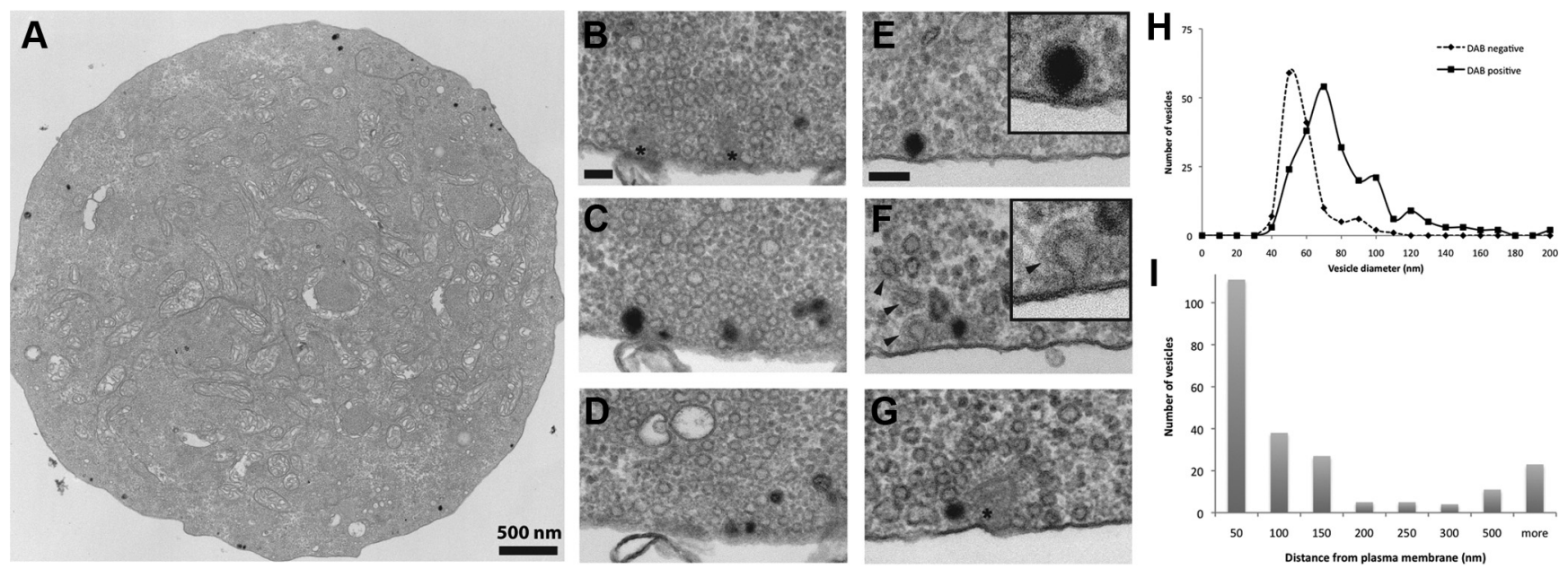

Figure 1. HRP labeling of endocytic structures corresponding to the fast pathway in bipolar cell terminals following $20 \mathrm{~ms}$ depolarizations. $A$, A complete single section of a bipolar cell terminal (enlarged version shown in supplemental Figure S1, available at www.jneurosci.org as supplemental material) after five $20 \mathrm{~ms}$ depolarizations showing HRP label incorporated into structures. The overall morphology demonstrated is representative of at least 5 independent presynaptic terminal serial section EM analyses. $\boldsymbol{B}-\boldsymbol{D}$, Serial sections of an enlarged area showing label is incorporated into vesicles. $\boldsymbol{E}-\boldsymbol{G}$, Labeled vesicles are frequently close to the membrane $(\boldsymbol{E})$ and can also be found in the specialized release apparatus, the ribbon (G). Unlabeled coated structures (possibly clathrin-coated) are indicated by the arrowheads $(\boldsymbol{F})$. The solid structures that are smaller than vesicles are probably glycogen granules. Asterisks show position of ribbon structures. $S$ cale bars, 100 $\mathrm{nm}$. $\boldsymbol{H}$, Distribution of unlabeled vesicle diameters and HRP labeled vesicle diameters ( 224 structures from two presynaptic terminals). The average \pm SD for unlabeled was $54 \pm 11 \mathrm{~nm}$ and for labeled was $77 \pm 28 \mathrm{~nm}$. Because unlabeled vesicles are more difficult to define and we measured $\sim 5$ small unlabeled structures that were neighboring each labeled vesicles. $I$, Distribution of $\mathrm{HRP}$ labeled vesicle distances away from the plasma membrane. The quantifications represent an average of two terminals.

this is the case, we used the fluid phase marker HRP to label the endocytic structures formed during a train of five, $20 \mathrm{~ms}$ depolarizations delivered at intervals of $10 \mathrm{~s} \mathrm{(Fig.} 1 \mathrm{~A}$; supplemental Experimental Procedures, available at www.jneurosci.org as supplemental material). This stimulus was chosen because it is followed almost exclusively by fast endocytosis with a time constant of 1-2 s (von Gersdorff and Matthews, 1994; Neves and Lagnado, 1999). Cells were fixed within seconds of the end of this stimulus train, i.e., within $\sim 40 \mathrm{~s}$ of the first brief depolarizing step. Serial sections demonstrated that labeled compartments were small- to medium-sized vesicles, and not the larger tubules or cisternae formed by bulk endocytosis (Holt et al., 2003, 2004; Coggins et al., 2007) (Fig. $1 B-D$ ). Although this observation might be interpreted as reflecting the very rapid breakup of large endocytic compartments, this seems unlikely because previous studies of the ribbon synapse of bipolar cells have found that large compartments only accumulate after tens of seconds of continuous depolarization (Holt et al., 2003; Coggins et al., 2007), and breakup on the time scale of tens of minutes (Coggins et al., 2007), while we only depolarized terminals for a total time of $100 \mathrm{~ms}$ and fixed immediately.

Some HRP-labeled structures displayed an irregular surrounding density (Fig. 1E), but these do not appear to have an organized clathrin coat. However, given the paucity of coated vesicles found, it could be that many became uncoated during fixation. Occasionally, buds and vesicles with a regular coat, characteristic of clathrin, were observed close to the limiting membrane (Fig. $1 F$, arrowheads). Morphologically, these are endocytic intermediates, the lack of HRP labeling indicating that fission had not yet occurred (the electron-dense precipitate will not form using our protocol if the vesicles retain a link to the outside). Some of the HRP-positive vesicles were in the juxtamembrane position of the electron-opaque ribbon, a position thought to supply the first vesicles for exocytosis (Fig. $1 G)$. This suggests that both vesicle exocytosis and fast endocytosis occurred at, or very close to, the active zone for the majority of vesicles released during stimulation.
The mean diameter of labeled vesicles was $77 \pm 28 \mathrm{~nm}$, while that of adjacent unlabeled vesicles was $54 \pm 11 \mathrm{~nm}$, with the two distributions overlapping considerably (Fig. $1 H$ ). The larger average diameter of labeled vesicles might be an effect of the precipitated DAB-HRP product, such as preventing shrinkage during fixation (Zuber et al., 2005). Most HRP-positive structures were found in the periphery of the cell; $50 \%$ were $<50 \mathrm{~nm}$ from the plasma membrane, and $81 \%$ within $200 \mathrm{~nm}$, while unstimulated cells did not contain any detectable HRP product (Fig. $1 I$ ). Altogether, these results indicate the preference of newly formed vesicles to be located in the submembranous region, with some in the vicinity of ribbons (Fig. $1 G$ ). The HRP labeling was very efficient: for example, in a single electron microscopy experiment, 1895 vesicles were labeled per terminal after the delivery of five brief $(20 \mathrm{~ms})$ depolarizing steps, corresponding to a membrane surface area of $\sim 40 \mu \mathrm{m}^{2}$ with an expected total capacitance of $320 \mathrm{fF}$. This figure agrees with the measured capacitance of endocytosed membrane during such a stimulus train, which is $\sim 150-300 \mathrm{fF}$ (Gomis et al., 1999). Retrieval into small vesicles after a short stimulus contrasts with the effects of chronic (1 min) stimulation, when $90 \%$ of the excess membrane is retrieved into large cisternae, each with an average membrane area 10 times that of a small vesicle (Holt et al., 2003, 2004). We therefore conclude that fast endocytosis in bipolar cells occurs predominantly into compartments approximating the size and shape of synaptic vesicles (with some being attached to ribbons and therefore being defined as synaptic vesicles), rather than the larger cisternae that have been observed after chronic stimulation (Paillart et al., 2003; Holt et al., 2004; Coggins et al., 2007).

\section{Capacitance measurements distinguish two molecularly distinct modes of endocytosis-fast and slow}

A number of studies using the capacitance technique have demonstrated the existence of two kinetically distinct modes of vesicle retrieval in the synaptic terminal of retinal bipolar cells (von Gersdorff and Matthews, 1994; Neves and Lagnado, 1999; Heidelberger et al., 2002; Hull and von Gersdorff, 2004). Follow- 
Table 1. Properties of the capacitance responses

\begin{tabular}{|c|c|c|c|c|c|}
\hline & $\Delta C_{\mathrm{m}}(\mathrm{fF})$ & $A_{\text {fast }}(\%)$ & $A_{\text {slow }}(\%)$ & $k_{\text {fast }}\left(s^{-1}\right)$ & $k_{\text {slow }}\left(s^{-1}\right)$ \\
\hline \multicolumn{6}{|l|}{20 ms stimulus } \\
\hline Control $(n=10)$ & $47 \pm 6$ & $98 \pm 3$ & $<5 \%$ & $0.8 \pm 0.1$ & NA \\
\hline PP19 peptide $(n=6)$ & $61 \pm 8$ & $64 \pm 15$ & No slow phase & $0.9 \pm 0.15$ & NA \\
\hline Endophilin $\Delta \mathrm{SH} 3(n=7)$ & $72 \pm 8$ & $49 \pm 11^{*}$ & $51 \pm 11$ & $1.1 \pm 0.2$ & $0.04 \pm 0.02$ \\
\hline \multicolumn{6}{|l|}{$100 \mathrm{~ms}$ stimulus } \\
\hline Control $(n=14)$ & $80 \pm 13$ & $61 \pm 7$ & $39 \pm 7$ & $1.2 \pm 0.3$ & $0.08 \pm 0.02$ \\
\hline PP19 peptide $(n=7)$ & $69 \pm 11$ & $51 \pm 8$ & No slow phase & $0.79 \pm 0.12$ & NA \\
\hline Endophilin $\Delta$ SH3 $(n=17)$ & $71 \pm 12$ & No fast phase ${ }^{*}$ & 100 & NA & $0.06 \pm 0.02$ \\
\hline Endophilin $\Delta$ N-Helix $\Delta$ SH3 $(n=8)$ & $90 \pm 26$ & $65 \pm 9$ & $35 \pm 9$ & $0.9 \pm 0.1$ & $0.08 \pm 0.01$ \\
\hline Amphiphysin $\Delta \mathrm{SH} 3(n=6)$ & $116 \pm 23$ & $63 \pm 9$ & $47 \pm 9$ & $1.2 \pm 0.2$ & $0.07 \pm 0.01$ \\
\hline \multicolumn{6}{|l|}{2 s stimulus } \\
\hline Control $(n=11)$ & $137 \pm 27$ & $11 \pm 7$ & $89 \pm 7$ & 0.47 & 0.16 \\
\hline Endophilin $\Delta \mathrm{SH} 3(n=8)$ & $145 \pm 30$ & No fast phase & 100 & NA & 0.11 \\
\hline
\end{tabular}

The amount of exocytosis is measured as the amplitude of the capacitance response $\left(\Delta C_{m}\right)$ (in femto-farads) immediately after calcium channels are closed. The amplitudes of the fast and slow phases $\left(A_{\mathrm{fast}}\right.$ and $\left.A_{\mathrm{slow}}\right)$, and rate constants $\left(k_{\text {fast }}\right.$ and $\left.k_{\text {slow }}\right)$ were obtained from double- or single-exponential fits, as described in supplemental material (available at www.jneurosci.org), but where there is no slow or no fast phase noted in the table, then the trace has been fitted with a single exponential. All data are expressed as mean \pm SEM Entries in bold are significantly different from the other conditions using a one-way ANOVA followed by Dunnett's multiple-comparisons test ( $p<0.01$ ).

*Slope of the initial retrieval: control, $30 \pm 2 \% \mathrm{~s}^{-1}$; endophilin $\Delta \mathrm{SH} 3, \mathbf{6} \pm 2 \% \mathrm{~s}^{-1}$.

ing exocytosis triggered by a $100 \mathrm{~ms}$ depolarization, all excess membrane is retrieved, and the time course is described by the sum of two declining exponentials (supplemental Fig. S2, available at www.jneurosci.org as supplemental material; Table 1). Fast endocytosis and slow endocytosis have time constants of $\sim 1-2 \mathrm{~s}$ and 10-20 s, respectively, and Jockusch et al. (2005) have shown that these kinetic phases display important molecular differences. We now repeat one such experiment (supplemental Fig. S2, available at www.jneurosci.org as supplemental material). The purple trace shows the effects of dialyzing an amphiphysin peptide (Amph-DNF) into the terminals, which effectively inhibits the function of the clathrin adaptor protein complex AP2 (Olesen et al., 2008). Slow endocytosis is blocked, but the amplitude and rate of the fast phase of retrieval are unaffected. A selective inhibition of slow endocytosis was also observed after introducing a peptide derived from the region of amphiphysin that binds dynamin, or the $\mathrm{N}$-terminal domain of clathrin heavy chain that binds accessory proteins, or a peptide derived from amphiphysin that binds clathrin (Jockusch et al., 2005). Thus, fast endocytosis is proposed to occur by a mechanism that is distinct from the classical clathrin-AP2-dependent pathway. It is also striking that inhibition of clathrin-AP2-mediated endocytosis leaves the membrane normally retrieved by this mechanism "stranded" at the surface (the capacitance trace in supplemental Fig. S2, available at www.jneurosci.org as supplemental material, does not return to baseline after the fast phase of endocytosis is complete); the fast mechanism of endocytosis cannot compensate for the loss of the slower clathrin-dependent mechanism.

\section{An endophilin SH3 domain-binding peptide inhibits both the fast and slow phases of endocytosis, but also targets the intersectin $\mathrm{SH} 3$ domain $\mathrm{A}$}

We set out to investigate the role of endophilin in synaptic vesicle endocytosis. Endophilin contains an N-BAR domain that curves membranes and would be predicted to act in a pathway where retrieval occurs via small vesicles (Gallop et al., 2006). The protein is implicated in clathrin-mediated endocytosis and in retrieval of synaptic vesicles, but its precise role is unclear (Guichet et al., 2002; Verstreken et al., 2002; Fabian-Fine et al., 2003; Schuske et al., 2003; Dickman et al., 2005). We first verified by Western blotting and immunofluorescence the presence of endophilin, and its major binding partners synaptojanin and dynamin, in goldfish brain and bipolar cells (Fig. $2 \mathrm{~A}$; supplemental Fig. S3, available at www.jneurosci.org as supplemental mate- rial). Endophilin is enriched in the synaptic terminal, in particular, to the peripheral region occupied by synaptic vesicles (Fig. $2 \mathrm{~A}$ compared with Fig. $1 A$ ).

To test the involvement of endophilin in the fast mode of endocytosis, we introduced PP19, a peptide from synaptojanin that interacts with endophilin SH3 domain (Ringstad et al., 1999), and measured its effect on the kinetics of endocytosis (Fig. $2 \mathrm{~B}, \mathrm{C})$. Under control conditions, a $20 \mathrm{~ms}$ stimulus was followed by fast endocytosis with a rate constant $k_{\text {fast }}=0.8 \mathrm{~s}^{-1}$ (i.e., $t_{\text {fast }}=$ $1.25 \mathrm{~s}$ ), which retrieved $\sim 98 \%$ of the excess membrane. Thus, recovery after a brief stimulus was dominated by fast endocytosis, as described previously (Neves and Lagnado, 1999; Hull and von Gersdorff, 2004). But when the patch pipette contained $100 \mu \mathrm{M}$ PP19, only $64 \%$ of the excess membrane was retrieved, and the remainder appeared "stuck" at the surface (Fig. $2 B$ ). The rate constant of recovery, $0.9 \mathrm{~s}^{-1}$, was within the range defining fast endocytosis (Neves and Lagnado, 1999; Hull and von Gersdorff, 2004). The essential effect of PP19, therefore, was to cause a block of fast retrieval for a proportion of the vesicles released by a brief stimulus.

The effects of PP19 were distinct from those of other interventions known to alter the kinetics of endocytosis in this synaptic terminal. The proportion of membrane retrieved by slow endocytosis increases when more vesicles are released, when calcium buffers are introduced into the terminal (Neves et al., 2001), and when the internal chloride concentration is elevated (Hull and von Gersdorff, 2004). It seems, therefore, that the slow mechanism, which is clathrin-AP2-dependent (Jockusch et al., 2005), is the default mechanism that operates when fast endocytosis is antagonized or its capacity exceeded. In contrast, a slow phase of endocytosis did not occur in the presence of PP19, and a proportion of the excess membrane was not recovered (Fig. $2 \mathrm{~B}$ ), indicating that the peptide had a second effect: a complete block of slow endocytosis. To investigate this idea further, we also tested the effect of PP19 after a longer ( $100 \mathrm{~ms})$ stimulus, when a greater proportion of the excess membrane is retrieved by slow endocytosis (Fig. 2C). In the presence of PP19, 51\% of the excess membrane was retrieved with a rate constant of $0.79 \mathrm{~s}^{-1}$, and the remainder was still not retrieved $20 \mathrm{~s}$ after the stimulus. The slow mode of endocytosis was again completely blocked. Proper interaction of SH3 binding partners was therefore important for both fast and slow endocytosis.

We wondered how specific PP19 was for endophilin relative to other SH3 domains, particularly those of intersectin, for which 
synaptojanin is also a major binding partner. In GST-SH3 domain cosedimentation assays (Fig. 2D), synaptojanin and dynamin interactions with endophilin and intersectin SH3A were both weakened by PP19. The effect of PP19 on binding to other $\mathrm{SH} 3$ domains is shown in supplemental Figure S3 (available at www.jneurosci.org as supplemental material). Thus, we could not be confident that PP19 acted selectively on endophilin.

\section{Dominant-negative endophilin inhibits} synaptojanin recruitment to liposomes Because of the lack of specificity of PP19, we sought an alternative way to acutely disrupt the function of endophilin, and predicted that endophilin lacking its $\mathrm{SH} 3$ domain might act as a dominant-negative inhibitor (a diagram of endophilin is shown in Fig. $3 A$ ). This molecule is unable to bind synaptojanin or dynamin and is expected to occupy normal endophilin-binding sites on the membrane, or heterodimerize with endogenous endophilin, and thus reduce the effective concentration of endophilin $\mathrm{SH} 3$ domains present on the membrane, abrogating synaptojanin and dynamin recruitment. We tested the effectiveness of endophilin $\Delta \mathrm{SH} 3$ as a dominant-negative construct in vitro in synaptojanin recruitment to liposomes and found that addition of endophilin $\Delta \mathrm{SH} 3$ in trans competed out the binding of synaptojanin and endophilin to liposomes (Fig. 3C).

\section{Dominant-negative endophilin selectively inhibits fast endocytosis in bipolar cells}

Endophilin $\Delta \mathrm{SH} 3$ was introduced into the synaptic terminal of bipolar cells through the patch pipette, at concentrations of 5, 12.5, and $25 \mu \mathrm{M}$. After a $100 \mathrm{~ms}$ depolarization, endocytosis normally occurred in two distinct phases, with $\sim 60 \%$ of the membrane retrieved rapidly and the remainder slowly (Fig. $4 A$ ). But upon introduction of endophilin $\Delta \mathrm{SH} 3$ (N-BAR), membrane retrieval was monophasic, with a time constant (16 s) characteristic of slow endocytosis (Neves et al., 2001; Hull and von Gersdorff, 2004). The fast mode of endocytosis was no longer evident (Fig. 4A, Table 1). The effects of endophilin $\Delta \mathrm{SH} 3$ were concentration dependent and inhibition was observed within a few minutes of beginning dialysis, with pipette concentrations $>12.5 \mu \mathrm{M}$. Run-down of endocytosis contributed minimally to this observation (supplemental Fig. S4, available at www.jneurosci.org as supplemental material). By calculating the time course over which endophilin $\Delta \mathrm{SH} 3$ accumulated in the terminal, we estimated that half-maximal inhibition of fast endocytosis occurred at $\sim 7 \mu \mathrm{M}$ (supplemental Methods and supplemental Figure S5, available at www.jneurosci.org as supplemental material), close to the dimerization constant of endophilin (Gallop et al., 2006).

The lack of fast retrieval after dialysis with endophilin $\Delta \mathrm{SH} 3$ (Fig. $4 A$ ) could be due to a block in the fast pathway compensated for by the slow pathway, or a slowing down of the fast pathway making the rate constant indistinguishable from slow endocytosis. We attempted to distinguish these two scenarios by investi- gating fast endocytosis alone, by delivering briefer stimuli (Neves and Lagnado, 1999). Figure $4 B$ shows the capacitance response to a depolarization lasting $20 \mathrm{~ms}$, after which almost all vesicles were normally retrieved with a time constant of $\sim 1$ s (Table 1 ). If endophilin $\Delta \mathrm{SH} 3$ acted by slowing this fast mechanism, the fall in membrane area would be expected to remain monophasic but with a slower rate constant. Instead, we observed two distinct phases of retrieval. Approximately 50\% of the excess membrane was retrieved with a time constant $(0.9 \mathrm{~s})$ characteristic of fast endocytosis, while the remainder was retrieved slowly. An ANOVA test confirmed that the amplitude of the fast phase was significantly reduced by endophilin $\Delta \mathrm{SH} 3(p<0.01)$. The effect of endophilin $\Delta \mathrm{SH} 3$ was not, therefore, to uniformly slow down the rate of endocytosis, but to cause a proportion of vesicles to be retrieved by the slow mechanism rather than the fast.

A switch from fast to slow endocytosis has also been observed in the presence of excess calcium buffers (Neves et al., 2001) or increased internal chloride concentration (Hull and von Gersdorff, 2004). It seems that the slow pathway can compensate for block of fast endocytosis. Together with the effects of endophilin $\Delta \mathrm{SH} 3$, all these experiments indicate that slow clathrin-dependent retrieval is the default pathway for vesicles that are not recaptured by the fast (endophilin-dependent) mechanism.

If endophilin $\Delta \mathrm{SH} 3$ selectively antagonizes the fast mode of endocytosis, then it should have no effect on the fall in membrane capacitance when this normally occurs by slow endocytosis. To test this, we used stimuli lasting $2 \mathrm{~s}$, when almost all the vesicles are normally retrieved slowly (Fig. $4 C$ ). Endophilin $\Delta \mathrm{SH} 3$ had no significant effect on the rate of membrane retrieval after this stim- 

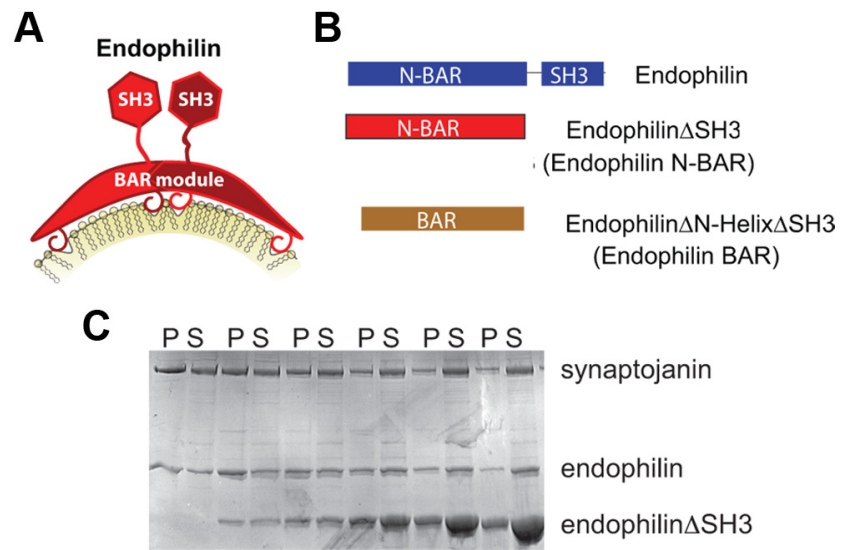

Figure 3. Endophilin $\Delta S H 3$ blocks recruitment of synaptojanin to liposomes. $A$, Diagram of endophilin binding to membranes. The BAR domain is responsible for membrane binding dimerization and curvature sensing. An amphipathic helix and the $\mathrm{N}$ terminus inserts into the membrane like a wedge helping to drive positive membrane curvature. $\boldsymbol{B}$, Domain structure of endophilin. The SH3 domain binds to dynamin and synaptojanin. $\mathbf{C}$, Endophilin $\Delta \mathrm{SH} 3$ inhibits synaptojanin recruitment to membranes. Endophilin was incubated with liposomes to enable synaptojanin recruitment (proteins are from rat and have $75 \%$ and $73 \%$ identity to zebrafish orthologs). Bound protein was separated from unbound in a liposome cosedimentation assay. Endophilin $\Delta \mathrm{SH} 3$ was then titrated into the experiment, where it can heterodimerize with full-length endophilin (with its $\mathrm{SH} 3$ domain), and thus compete with synaptojanin binding. P, Pellet; $S$, supernatant.

ulus, providing further evidence that this construct did not affect the slow, clathrin-AP2-dependent, pathway of retrieval. The shift from fast to slow modes of endocytosis on introducing the endophilin $\Delta \mathrm{SH} 3$ did not correlate with any obvious change in the amount of exocytosis (Table 1) or the amplitudes of the $\mathrm{Ca}^{2+}$ currents.

\section{The block of fast endocytosis by endophilin $\Delta \mathrm{SH} 3$ is specific} and requires membrane localization

Endophilin $\Delta \mathrm{SH} 3$ can tubulate membranes in vitro, much like other proteins containing N-BAR domains (supplemental Fig. S6, available at www.jneurosci.org as supplemental material), so it was important to test the specificity of its effect. Dialysis with 25 $\mu \mathrm{M}$ Drosophila amphiphysin $\Delta \mathrm{SH} 3$, also a potent tubulator (a muscle enriched protein), had no significant effect on the fast or slow phases of endocytosis observed after a 20 or $100 \mathrm{~ms}$ depolarization (Fig. $5 A, B$ ). The efficient targeting of endophilin to membranes in vitro requires the $\mathrm{N}$-terminal amphipathic helix (Gallop et al., 2006). Therefore, we introduced the endophilin BAR domain alone into terminals. This construct had no effect on retrieval (Fig. $5 \mathrm{C}$ ), indicating that the competitive interaction with endogenous endophilin occurred at the membrane. In contrast to endophilin $\Delta \mathrm{SH} 3$, fast endocytosis was maintained even after $\sim 4$ min of dialysis with the endophilin BAR domain alone. Full-length endophilin retaining the SH3 domain mildly inhibited both fast and slow phases of endocytosis in the synaptic terminal (Fig. $5 D$ ), possibly by competing with binding partners. Finally, the BAR domain of arfaptin2, which targets Golgi membranes, did not inhibit vesicle retrieval (supplemental Fig. S7, available at www.jneurosci.org as supplemental material).

\section{Discussion}

We find that molecular interventions targeting endophilin and its binding partners inhibit fast endocytosis at the ribbon synapse of retinal bipolar cells. In contrast, inhibitors of AP2 and clathrin inhibited only slow endocytosis (Jockusch et al., 2005). Endophi-
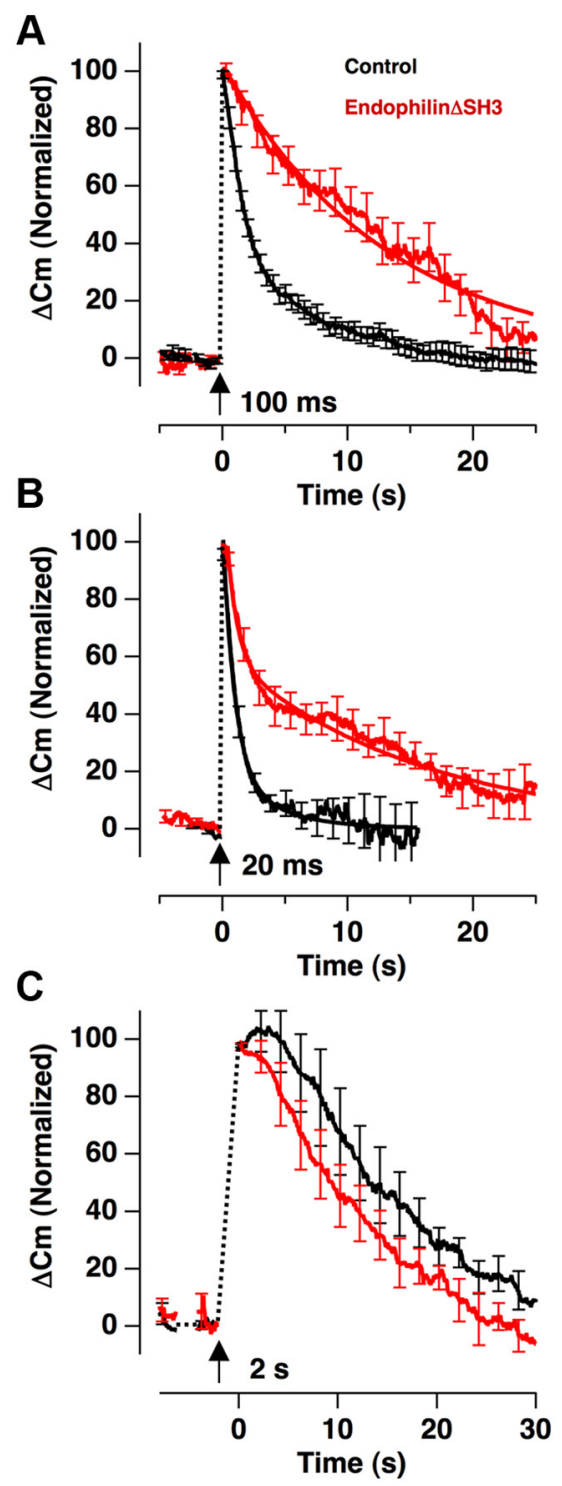

Figure 4. Endophilin $\Delta S H 3$ blocks fast endocytosis in bipolar cells. $A$, Endophilin $\Delta S H 3$ domain inhibits fast endocytosis in the synaptic terminal of retinal bipolar cells. Averaged capacitance responses to depolarizations of $100 \mathrm{~ms}$ delivered under control conditions (black, $n=$ 14) and after dialysis with $12.5-25 \mu \mathrm{m}$ endophilin $\Delta \mathrm{SH} 3$ (red, $n=7$; described as a single exponential with $k_{\text {slow }}=0.08 \mathrm{~s}^{-1}$ ). Error bars show 1 SEM. Amplitudes and time constants are shown in Table 1. Individual traces at various times after whole-cell recording are shown in supplemental Figure S5 (available at www.jneurosci.org as supplemental material). The concentration dependence of the endophilin $\Delta \mathrm{SH} 3$ inhibition is shown in supplemental Figure $\mathrm{S} 6$ (available at www.jneurosci.org as supplemental material). $\boldsymbol{B}$, Averaged capacitance responses to depolarizations of $20 \mathrm{~ms}(n=11)$. Control (black) is described as a double exponential with $A_{\text {fast }}=87 \%, k_{\text {fast }}=1.1 \mathrm{~s}^{-1}, k_{\text {slow }}=0.25 \mathrm{~s}^{-1}$. Endophilin $\Delta S H 3$ (red) is described with $A_{\text {fast }}=39 \%, k_{\text {fast }}=1.23 \mathrm{~s}^{-1}, k_{\text {slow }}=0.06 \mathrm{~s}^{-1}$. As in A, ANOVA indicated that the amplitude of the fast phase was significantly reduced by endophilin $\Delta$ SH3. C, Responses to depolarizations of $2 \mathrm{~s}(n=8)$. Control is described as a single exponential with $k_{\text {slow }}=0.08 \mathrm{~s}^{-1}$. Endophilin $\Delta S H 3$ is described as a single exponential with $k_{\text {slow }}=0.11 \mathrm{~s}^{-1}$.

lin thus represents the first protein, other than the scission molecule dynamin, implicated in fast retrieval of synaptic vesicles. These results do not rule out the possibility that endophilin may also play some role in slow endocytosis at the ribbon synapse of bipolar cells. It is possible that the fast and slow phases have different sensitivity to the inhibitors. In addition, the endophilin SH3 domain interacts with receptors, e.g., the vesicular glutamate transporter (Voglmaier et al., 2006); thus, endophilin $\Delta$ SH3 may not be effectively targeted to sites of slow endocytosis. 

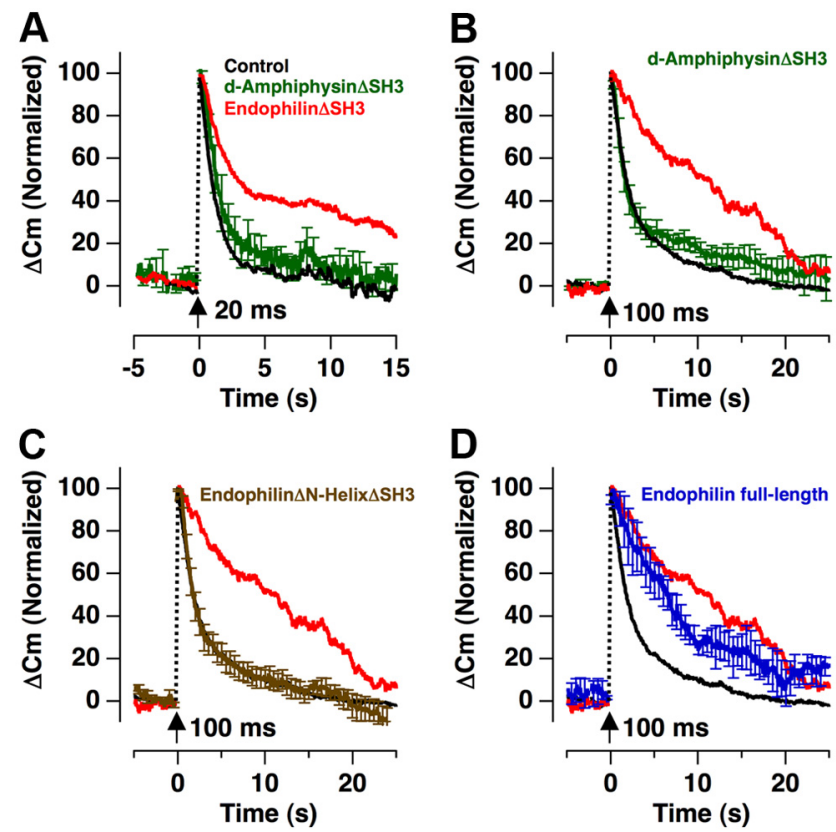

Figure 5. Control interventions amphiphysin $\Delta \mathrm{SH}$, endophilin $\Delta \mathrm{N}-\mathrm{Helix} \Delta \mathrm{SH} 3$, and fulllength endophilin do not block fast endocytosis in bipolar cells. Inhibition of the fast phase does not occur with amphiphysin N-BAR domain. $A$, Averaged capacitance responses to 20 ms depolarization after dialysis of $D$. melanogaster amphiphysin $\Delta \mathrm{SH} 3(25 \mu \mathrm{m}$, green, $n=3)$. Recovery is described as a single exponential with $A_{\text {fast }}=99 \%, k_{\text {fast }}=0.65 \mathrm{~s}^{-1}$. Traces from Figure $4 B$ are shown for comparison. $\boldsymbol{B}$, Averaged capacitance responses to $100 \mathrm{~ms}$ depolarization ( $25 \mu \mathrm{m}$, green, $n=5$ ). Recovery described as double exponential with $A_{\text {fast }}=65 \%, k_{\text {fast }}=0.88 \mathrm{~s}^{-1}$, $k_{\text {slow }}=0.07 \mathrm{~s}^{-1}$. In this and all subsequent panels, traces are shown superimposed on those from Figure 4A.C, Averaged capacitance responses obtained after dialysis with the endophilin BAR domain without the $\mathrm{N}$-terminal amphipathic helix (endophilin $\Delta \mathrm{N}$-helix $\Delta \mathrm{SH} 3$ : $12.5-25 \mu \mathrm{m}$, brown; $n=7$; single exponential, $\left.k_{\text {slow }}=0.11 \mathrm{~s}^{-1}\right) . D$, Averaged responses obtained after dialysis with full-length endophilin (12.5 $\mu \mathrm{m}$, blue; $n=4$; single exponential, $k_{\text {slow }}=0.12 \mathrm{~s}^{-1}$ ).

Endophilin has a clear role in synaptic vesicle retrieval, but has previously been assumed to act only in the clathrin-dependent pathway. This conclusion has been based on elevated numbers of clathrin-coated vesicles and budding profiles observed in endophilin mutant synapses (Guichet et al., 2002; Verstreken et al., 2002; Fabian-Fine et al., 2003; Schuske et al., 2003; Dickman et al., 2005). Such observations are also compatible with the idea that endophilin is involved in other endocytic mechanisms, since an increase in clathrin-coated membranes might also be caused by a compensatory upregulation of the clathrin-dependent pathway in response to inhibition of other pathways. Indeed, an important feature of our results was compensatory retrieval by slow endocytosis when the fast mechanism is inhibited (Figs. 4, 5). There are also indications that clathrin-dependent and clathrinindependent mechanisms can operate in synapses of C. elegans and D. melanogaster (Heerssen et al., 2008; Kasprowicz et al., 2008; Sato et al., 2009). In C. elegans, synaptic vesicle pools are maintained in temperature-sensitive clathrin mutants (unlike in endophilin mutants), and mutation of the sole $\mu$ subunit of AP2 shows unexpectedly modest effects (Gu et al., 2008; Sato et al., 2009).

The small size of vesicles endocytosed by the fast pathway confirms our previous molecular studies on endophilin, where we found that the N-BAR domain of endophilin generates or stabilizes membrane curvature and provides a link between membrane deformation and the recruitment of $\mathrm{SH} 3$ domain

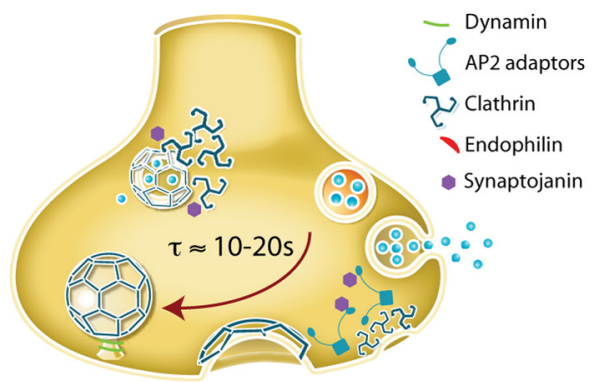

Slow endocytosis - clathrin dependent

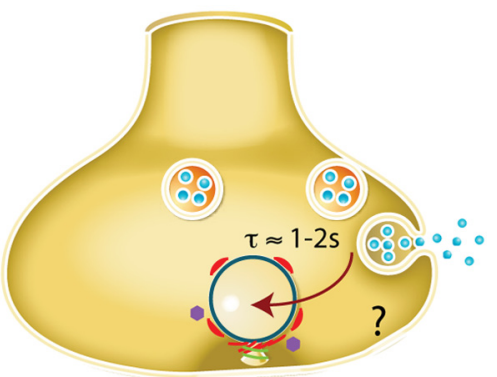

Fast endocytosis - endophilin dependent

Figure 6. Two endocytic pathways in the synaptic terminal of retinal bipolar cells. Recruitment of dynamin and synaptojanin in two endocytic pathways. Two modes of endocytosis, endophilin dependent (faster kinetics) and clathrin dependent (slower kinetics), operate in parallel, but are favored to different extents by the stimulus strength in retinal bipolar cell terminals. Endophilin may partially stabilize the vesicle curvature and/or aid the reinvagination after collapse. The speed of the fast pathway may arise from vesicle capture close to the site of fusion, as we frequently find labeled vesicles in the ribbon structures following short stimuli.

binding partners (Gallop et al., 2006). The fast time constant associated with the endophilin-dependent endocytic events implies that there is a short time window in which this protein must act before the vesicle is lost to this mechanism of retrieval. Due to its preloading onto synaptic vesicles (seen in C. elegans and D. melanogaster terminals and supported by its colocalization with synaptic vesicles in resting bipolar cell terminals; Fig. 2 A), endophilin is well placed to either rapidly generate curvature from a collapsed synaptic vesicle or to stabilize the curvature of a collapsing vesicle and encourage dynamin assembly and scission. Additionally, since synaptojanin is also a major binding partner of endophilin, this implicates a role for $\operatorname{PtdIns}(4,5) \mathrm{P}_{2}$ turnover in the fast pathway. This may be related to ribbon function since the synaptojanin zebrafish nrc mutant is unable to anchor ribbons (Van Epps et al., 2004), and there are several lines of evidence that fast retrieval is localized to ribbons: (1) DAB-positive vesicles appear in ribbons or nearby ribbons, (2) cell-attached capacitance recordings from active zones showed fast but not slow endocytosis (Llobet et al., 2003), and (3) longer stimulations evoke release outside of active zones, and vesicles are retrieved using the slow pathway.

To conclude, we propose that endophilin and its binding partners synaptojanin and dynamin are key components of a fast endocytic pathway that can be distinguished from classical clathrin-dependent retrieval by its kinetics and molecular mechanism (Fig. 6). It may be that fast and slow retrieval at ribbon synapses share common molecules other than dynamin, but this possibility remains to be investigated. 


\section{References}

Ceccarelli B, Hurlbut WP, Mauro A (1973) Turnover of transmitter and synaptic vesicles at the frog neuromuscular junction. J Cell Biol 57:499-524.

Coggins MR, Grabner CP, Almers W, Zenisek D (2007) Stimulated exocytosis of endosomes in goldfish retinal bipolar neurons. J Physiol 584:853-865.

Dickman DK, Horne JA, Meinertzhagen IA, Schwarz TL (2005) A slowed classical pathway rather than kiss-and-run mediates endocytosis at synapses lacking synaptojanin and endophilin. Cell 123:521-533.

Fabian-Fine R, Verstreken P, Hiesinger PR, Horne JA, Kostyleva R, Zhou Y, Bellen HJ, Meinertzhagen IA (2003) Endophilin promotes a late step in endocytosis at glial invaginations in Drosophila photoreceptor terminals. J Neurosci 23:10732-10744.

Gad H, Ringstad N, Löw P, Kjaerulff O, Gustafsson J, Wenk M, Di Paolo G, Nemoto Y, Crun J, Ellisman MH, De Camilli P, Shupliakov O, Brodin L (2000) Fission and uncoating of synaptic clathrin-coated vesicles are perturbed by disruption of interactions with the SH3 domain of endophilin. Neuron 27:301-312.

Gallop JL, Jao CC, Kent HM, Butler PJ, Evans PR, Langen R, McMahon HT (2006) Mechanism of endophilin N-BAR domain-mediated membrane curvature. EMBO J 25:2898-2910.

Gomis A, Burrone J, Lagnado L (1999) Two actions of calcium regulate the supply of releasable vesicles at the ribbon synapse of retinal bipolar cells. J Neurosci 19:6309-6317.

Gu M, Schuske K, Watanabe S, Liu Q, Baum P, Garriga G, Jorgensen EM (2008) Mu2 adaptin facilitates but is not essential for synaptic vesicle recycling in Caenorhabditis elegans. J Cell Biol 183:881-892.

Guichet A, Wucherpfennig T, Dudu V, Etter S, Wilsch-Bräuniger M, Hellwig A, González-Gaitán M, Huttner WB, Schmidt AA (2002) Essential role of endophilin A in synaptic vesicle budding at the Drosophila neuromuscular junction. EMBO J 21:1661-1672.

He L, Wu XS, Mohan R, Wu LG (2006) Two modes of fusion pore opening revealed by cell-attached recordings at a synapse. Nature 444:102-105.

Heerssen H, Fetter RD, Davis GW (2008) Clathrin dependence of synapticvesicle formation at the Drosophila neuromuscular junction. Curr Biol 18:401-409.

Heidelberger R, Zhou ZY, Matthews G (2002) Multiple components of membrane retrieval in synaptic terminals revealed by changes in hydrostatic pressure. J Neurophysiol 88:2509-2517.

Holt M, Cooke A, Wu MM, Lagnado L (2003) Bulk membrane retrieval in the synaptic terminal of retinal bipolar cells. J Neurosci 23:1329-1339.

Holt M, Cooke A, Neef A, Lagnado L (2004) High mobility of vesicles supports continuous exocytosis at a ribbon synapse. Curr Biol 14:173-183.

Hull C, von Gersdorff H (2004) Fast endocytosis is inhibited by GABAmediated chloride influx at a presynaptic terminal. Neuron 44:469-482.

Jockusch WJ, Praefcke GJ, McMahon HT, Lagnado L (2005) Clathrindependent and clathrin-independent retrieval of synaptic vesicles in retinal bipolar cells. Neuron 46:869-878.

Kasprowicz J, Kuenen S, Miskiewicz K, Habets RL, Smitz L, Verstreken P (2008) Inactivation of clathrin heavy chain inhibits synaptic recycling but allows bulk membrane uptake. J Cell Biol 182:1007-1016.

Llobet A, Cooke A, Lagnado L (2003) Exocytosis at the ribbon synapse of retinal bipolar cells studied in patches of presynaptic membrane. J Neurosci 23:2706-2714.

LoGiudice L, Matthews G (2007) Endocytosis at ribbon synapses. Traffic 8:1123-1128.

Neves G, Lagnado L (1999) The kinetics of exocytosis and endocytosis in the synaptic terminal of goldfish retinal bipolar cells. J Physiol 515:181-202.

Neves G, Gomis A, Lagnado L (2001) Calcium influx selects the fast mode of endocytosis in the synaptic terminal of retinal bipolar cells. Proc Natl Acad Sci U S A 98:15282-15287.

Olesen LE, Ford MG, Schmid EM, Vallis Y, Babu MM, Li PH, Mills IG, McMahon HT, Praefcke GJ (2008) Solitary and repetitive binding motifs for the AP2 complex alpha-appendage in amphiphysin and other accessory proteins. J Biol Chem 283:5099-5109.

Paillart C, Li J, Matthews G, Sterling P (2003) Endocytosis and vesicle recycling at a ribbon synapse. J Neurosci 23:4092-4099.

Rikhy R, Kumar V, Mittal R, Krishnan KS (2002) Endophilin is critically required for synapse formation and function in Drosophila melanogaster. J Neurosci 22:7478-7484.

Ringstad N, Gad H, Löw P, Di Paolo G, Brodin L, Shupliakov O, De Camilli P (1999) Endophilin/SH3p4 is required for the transition from early to late stages in clathrin-mediated synaptic vesicle endocytosis. Neuron 24: 143-154.

Rosenmund C, Stevens CF (1997) The rate of aldehyde fixation of the exocytotic machinery in cultured hippocampal synapses. J Neurosci Methods $76: 1-5$.

Sato K, Ernstrom GG, Watanabe S, Weimer RM, Chen CH, Sato M, Siddiqui A, Jorgensen EM, Grant BD (2009) Differential requirements for clathrin in receptor-mediated endocytosis and maintenance of synaptic vesicle pools. Proc Natl Acad Sci U S A 106:1139-1144.

Schuske KR, Richmond JE, Matthies DS, Davis WS, Runz S, Rube DA, van der Bliek AM, Jorgensen EM (2003) Endophilin is required for synaptic vesicle endocytosis by localizing synaptojanin. Neuron 40:749-762.

Stinchcombe JC, Nomoto H, Cutler DF, Hopkins CR (1995) Anterograde and retrograde traffic between the rough endoplasmic reticulum and the Golgi complex. J Cell Biol 131:1387-1401.

Van Epps HA, Hayashi M, Lucast L, Stearns GW, Hurley JB, De Camilli P, Brockerhoff SE (2004) The zebrafish nrc mutant reveals a role for the polyphosphoinositide phosphatase synaptojanin 1 in cone photoreceptor ribbon anchoring. J Neurosci 24:8641-8650.

Verstreken P, Kjaerulff O, Lloyd TE, Atkinson R, Zhou Y, Meinertzhagen IA, Bellen HJ (2002) Endophilin mutations block clathrin-mediated endocytosis but not neurotransmitter release. Cell 109:101-112.

Voglmaier SM, Kam K, Yang H, Fortin DL, Hua Z, Nicoll RA, Edwards RH (2006) Distinct endocytic pathways control the rate and extent of synaptic vesicle protein recycling. Neuron 51:71-84.

von Gersdorff H, Matthews G (1994) Inhibition of endocytosis by elevated internal calcium in a synaptic terminal. Nature 370:652-655.

Wu LG, Ryan TA, Lagnado L (2007) Modes of vesicle retrieval at ribbon synapses, calyx-type synapses, and small central synapses. J Neurosci 27:11793-11802.

Zuber B, Nikonenko I, Klauser P, Muller D, Dubochet J. (2005) The mammalian central nervous synaptic cleft contains a high density of periodically organized complexes. Proc Natl Acad Sci U S A 102:19192-19197. 